\title{
Chemical composition of the stellar cluster Gaia1: no surprise behind Sirius ${ }^{\star}$
}

\author{
A. Mucciarelli ${ }^{1,2}$, L. Monaco ${ }^{3}$, P. Bonifacio ${ }^{4}$, and I. Saviane ${ }^{5}$ \\ 1 Dipartimento di Fisica e Astronomia, Università degli Studi di Bologna, Viale Berti Pichat, 6/2, 40127 Bologna, Italy \\ e-mail: alessio.mucciarelli2@unibo.it \\ 2 INAF-Osservatorio Astronomico di Bologna, via Ranzani 1, 40127 Bologna, Italy \\ 3 Departamento de Ciencias Fisicas, Universidad Andres Bello, Fernandez Concha 700, Las Condes, Santiago, Chile \\ ${ }^{4}$ GEPI, Observatoire de Paris, PSL Research University, CNRS, Place Jule Janssen, 92190 Meudon, France \\ 5 European Southern Observatory, Alonso de Cordova 4860, Macul, Santiago, Chile
}

Received 19 April 2017 / Accepted 3 June 2017

\begin{abstract}
We observed six He-clump stars of the intermediate-age stellar cluster Gaial with the MIKE/Magellan spectrograph. A possible extra-galactic origin of this cluster, recently discovered thanks to the first data release of the ESA Gaia mission, has been suggested, based on its orbital parameters. Abundances for Fe, $\alpha$, proton- and neutron-capture elements have been obtained. We find no evidence of intrinsic abundance spreads. The iron abundance is solar $([\mathrm{FeI} / \mathrm{H}]=+0.00 \pm 0.01 ; \sigma=0.03 \mathrm{dex})$. All the other abundance ratios are generally solar-scaled, similar to the Galactic thin disk and open cluster stars of similar metallicity. The chemical composition of Gaia1 does not support an extra-galactic origin for this stellar cluster, which can be considered as a standard Galactic open cluster.
\end{abstract}

Key words. stars: abundances - techniques: spectroscopic - open clusters and associations: individual: Gaia1

\section{Introduction}

Gaia1 is a stellar cluster that has recently been identified by Koposov et al. (2017) using the first data release of the ESA Gaia mission (Gaia Collaboration 2016). An identification of this cluster has been precluded for decades by its proximity ( $\sim 10$ arcmin) to the bright star Sirius.

Recently, Simpson et al. (2017, hereafter S17) performed a prompt spectroscopic follow-up of this system, confirming that Gaial is a stellar cluster, according to its radial velocity (RV) and $[\mathrm{Fe} / \mathrm{H}]$. They identified 41 cluster members, 27 of them observed with the high-resolution spectrograph HERMES and the others with the low-resolution spectrograph AAOmega, both at the Anglo-Australian Telescope. The mean radial velocity is $+58.30 \pm 0.22 \mathrm{~km} \mathrm{~s}^{-1}$, with a dispersion of $0.94 \pm 0.15 \mathrm{~km} \mathrm{~s}^{-1}$, while the mean iron abundance (from HERMES targets alone) is $[\mathrm{Fe} / \mathrm{H}]=-0.13 \pm 0.13$ dex and its age is $\sim 3$ Gyr. Using Gaia and 2MASS positions for the cluster stars, S17 derived a first estimate of its proper motion and orbit, in particular, they found a Galactocentric distance $\left(R_{\mathrm{GC}}\right)$ of $11.8 \pm 0.2 \mathrm{kpc}$, a maximum height above the Galactic plane of $z_{\max }=1.7_{-0.9}^{+2.1} \mathrm{kpc}$, and an eccentricity $\epsilon=0.3 \pm 0.2$. These orbital parameters, taken at face value, could suggest an extra-galactic origin for Gaia1, even though they have large uncertainties that prevent any firm conclusion.

In this paper we present chemical abundances of $\mathrm{Fe}, \mathrm{Na}, \mathrm{Mg}$, $\mathrm{Al}, \mathrm{Si}, \mathrm{Ca}, \mathrm{Ti}, \mathrm{Ba}$, and $\mathrm{Eu}$ for six giant stars observed at the Magellan II Telescope.

\footnotetext{
* The full Table A.1 is only available at the CDS via anonymous ftp to cdsarc.u-strasbg. fr (130.79.128.5) or via http://cdsarc.u-strasbg.fr/viz-bin/qcat?J/A+A/603/L7
}

\section{Observations}

Spectra of seven stars with infrared colours and magnitudes compatible with being He-clump stars of the stellar cluster Gaia1 have been secured using the Magellan Inamori Kyocera Echelle (MIKE) spectrograph (Bernstein et al. 2003) mounted on the Magellan II Telescope at Las Campanas Observatory. Table 1 lists the coordinates, the 2MASS magnitudes, RVs, and atmospheric parameters for the observed targets. The position of the observed targets in the 2MASS $\left(K_{\mathrm{S}}\right.$ vs. $\left(J-K_{\mathrm{S}}\right)$ ) colour-magnitude diagram is shown in upper left panel of Fig. 1 as red points. The lower left panel shows (as blue points) the position of the targets observed with HERMES by S17. All the MIKE targets have been observed with the $0.7^{\prime \prime} \times 5.0^{\prime \prime}$ slit, providing a spectral resolution of $\sim 36000$ in the red arm (covering from $\sim 5000$ to $\sim 9150 \AA$ ). Exposure times varied from 600 to $1800 \mathrm{~s}$. The signal-to-noise ratio $(\mathrm{S} / \mathrm{N})$ ranges from $\sim 40$ to $\sim 80$ at $\sim 6000 \AA$ A. Bias-subtraction, flat-fielding, spectral extraction, and wavelength calibration have been performed using the CarPy MIKE pipeline (Kelson 2003).

Radial velocities have been obtained with DAOSPEC (Stetson \& Pancino 2008) by measuring the position of almost 400 metallic and unblended lines selected in the red spectrum. Six of the target stars share very similar RVs (see Table 1) and are classified as members of Gaia1. Star 3 presents a different RV and damped absorption lines, however, is therefore not considered a member and is not discussed further. The mean heliocentric RV is $+57.6 \pm 0.4 \mathrm{~km} \mathrm{~s}^{-1}\left(\sigma=1.0 \mathrm{~km} \mathrm{~s}^{-1}\right)$, which matches the value derived by $\mathrm{S} 17$ well. 
Table 1. Main information on the observed MIKE targets.

\begin{tabular}{|c|c|c|c|c|c|c|c|c|c|c|}
\hline ID & $\mathrm{ID}_{2 \mathrm{MASS}}$ & $\begin{array}{c}\mathrm{RA}_{J 2000} \\
\text { [deg] }\end{array}$ & $\begin{array}{c}\operatorname{Dec}_{J 2000} \\
{[\mathrm{deg}]}\end{array}$ & $J$ & $K_{\mathrm{S}}$ & $\begin{array}{c}\mathrm{RV} \\
{\left[\mathrm{km} \mathrm{s}^{-1}\right]}\end{array}$ & $\begin{array}{c}T_{\mathrm{eff}} \\
{\left[{ }^{o} \mathrm{~K}\right]}\end{array}$ & $\begin{array}{c}\log g \\
{\left[\mathrm{~cm} \mathrm{~s}^{-2}\right]}\end{array}$ & $\begin{array}{c}v_{\mathrm{t}} \\
{\left[\mathrm{km} \mathrm{s}^{-1}\right]}\end{array}$ & $\begin{array}{c}S / N \\
\text { at } 600 \mathrm{~nm}\end{array}$ \\
\hline 1 & 2MASS 06455819-1641596 & 101.492499 & -16.699909 & 12.731 & 11.860 & +58.2 & $5000 \pm 60$ & $2.68 \pm 0.10$ & $1.5 \pm 0.1$ & 60 \\
\hline 2 & 2MASS 06454837-1643113 & 101.451559 & -16.719822 & 12.782 & 11.932 & +58.4 & $4960 \pm 80$ & $2.67 \pm 0.12$ & $1.5 \pm 0.1$ & 40 \\
\hline 3 & 2MASS 06454801-1642240 & 101.450069 & -16.706678 & 12.832 & 11.996 & +64.5 & - & - & - & 40 \\
\hline 4 & 2MASS 06455237-1643471 & 101.468234 & -16.729773 & 12.698 & 11.878 & +57.2 & $5050 \pm 80$ & $2.70 \pm 0.12$ & $1.4 \pm 0.1$ & 40 \\
\hline 5 & 2MASS 06455379-1645521 & 101.474151 & -16.764490 & 12.770 & 11.930 & +57.4 & $4960 \pm 60$ & $2.67 \pm 0.10$ & $1.4 \pm 0.1$ & 70 \\
\hline 6 & 2MASS 06460723-1647294 & 101.530159 & -16.791500 & 12.753 & 11.913 & +56.0 & $4920 \pm 50$ & $2.66 \pm 0.10$ & $1.3 \pm 0.1$ & 80 \\
\hline 7 & 2MASS 06455253-1640582 & 101.468910 & -16.682854 & 12.758 & 11.918 & +58.5 & $4960 \pm 50$ & $2.67 \pm 0.10$ & $1.6 \pm 0.1$ & 80 \\
\hline
\end{tabular}

Notes. Columns are: 2MASS identification number, 2MASS $J$ and $K_{\mathrm{S}}$ magnitudes, RV, and atmospheric parameters derived from the MIKE spectra, together with the $\mathrm{S} / \mathrm{N}$ measured at $\sim 600 \mathrm{~nm}$.
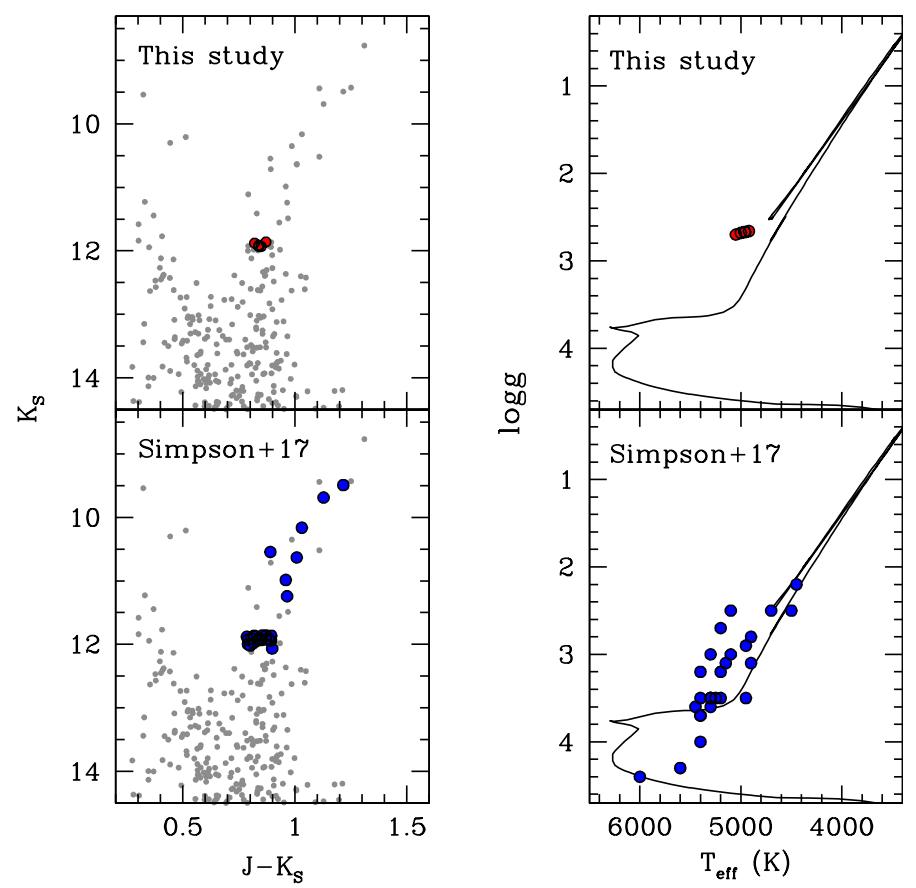

Fig. 1. Left panels: $2 \mathrm{MASS}\left(K_{\mathrm{S}}\right.$ vs. $\left.\left(J-K_{\mathrm{S}}\right)\right)$ colour-magnitude diagram of Gaia1 (grey points) with the position of our targets (red points) and those of S17 (blue points). Right panels: position of the MIKE and HERMES targets in the $T_{\text {eff }}-\log g$ plane, superimposed on a theoretical isochrone by Bressan et al. (2012) with $[\mathrm{Fe} / \mathrm{H}]=0.0$ and age $3 \mathrm{Gyr}$. Unexpectedly, the S17 stars do not define an RGB in the theoretical plane, suggesting that their parameters are not correct.

\section{Chemical analysis}

\subsection{Atmospheric parameters}

The atmospheric parameters were derived as follows: (i) effective temperatures $\left(T_{\text {eff }}\right)$ were derived spectroscopically from the excitation equilibrium based on the large number $(\sim 120)$ of measured Fe I lines; (ii) surface gravities $(\log g)$ were obtained from the Stefan-Boltzmann relation, adopting the spectroscopic $T_{\text {eff }}$, the bolometric corrections calculated according to Buzzoni et al. (2010), the true distance modulus $(m-M)_{0}=13.3(\mathrm{~S} 17$ from the 2MASS photometry), and the colour excess $E(B-V)=0.41 \mathrm{mag}$ obtained from the maps by Schlegel et al. (1998) corrected according to Bonifacio et al. (2000b). For all the stars a stellar mass of $1.5 M_{\odot}$ was adopted; (iii) microturbulent velocities $\left(v_{t}\right)$ were obtained by minimizing the trend between iron abundance and line strength.

The advantage of this hybrid approach is that all the information at hand from spectroscopy and photometry is exploited best, and uncertainties in the colour excess that mainly affect the photometric $T_{\text {eff }}$ are minimized. We note that the uncertainty in the colour excess marginally affects the determination of $\log g$. The values of $E(B-V)$ in the direction of Gaial range from 0.36 to $0.66 \mathrm{mag}$ (see $\mathrm{S} 17$ and references therein). A variation of \pm 0.1 mag in $E(B-V)$ leads to a variation in $\log g$ of \pm 0.002 , but has a significant $( \pm 140 \mathrm{~K})$ effect on $T_{\text {eff }}$ even when they are derived from a colour that is only marginally affected by the reddening, such as $\left(J-K_{\mathrm{S}}\right)$. Possible systematics in the ionization equilibrium are also minimized. These are due to over-ionization effects that affect the spectroscopic $\log g$.

The upper right panel of Fig. 1 shows the position of the observed targets in the $T_{\text {eff }}-\log g$ plane (red points) in comparison with a theoretical isochrone from the Padua database (Bressan et al. 2012) with solar metallicity and an age of $3 \mathrm{Gyr}$ (see S17). We find that within the uncertainties, the atmospheric parameters (derived as explained above) fit the position expected for the He-clump reasonably well. On the other hand, an inspection on the atmospheric parameters derived by S17 for their targets reveals a significant discrepancy with the atmospheric parameters expected for their evolutionary stage (lower right panel of Fig. 1). Even though their observed targets belong to the $\mathrm{He}-$ clump or to the bright red giant branch, $T_{\text {eff }}$ and $\log g$ are compatible with those of less evolved stars. In particular, half of their sample (14 out 27 stars) have $\log g$ higher than 3.5, which are unlikely values for the observed targets, and a systematic offset toward higher $T_{\text {eff }}$ seems to be present, with the extreme case of a He-clump star for which they estimated $T_{\text {eff }}=6000 \mathrm{~K}$ and $\log g=4.4$. We note that S17 derived all the atmospheric parameters spectroscopically: we stress that this approach can be very misleading in case of low-quality spectra and/or when the number of $\mathrm{Fe}$ lines are not large enough to guarantee a robust coverage in excitation potential and line strength, or when there are too few or too weak Fe II lines.

\subsection{Sanity check}

As a sanity check, we derived the atmospheric parameters using different methods. First, $T_{\text {eff }}$ and $\log g$ were derived from the photometry, adopting the $(J-K)_{0}-T_{\text {eff }}$ transformation by Gonzalez Hernandez \& Bonifacio (2009). The photometric $T_{\text {eff }}$ are on average lower than the spectroscopic ones by $\sim 200-300 \mathrm{~K}$. However, a significant (at a level of $3-5 \sigma$ ) positive slope between iron abundances and excitation potential is found for all the targets, which indicates that the photometric $T_{\text {eff }}$ are not entirely correct, probably because of the large uncertainties in the colour excess. The use of photometric parameters leads to a decrease in $[\mathrm{Fe} / \mathrm{H}]$ of about 0.2 dex. On the other hand, a fully spectroscopic determination of all the parameters provides 
a $T_{\text {eff }}$ that is very similar to that obtained above and $\log g$ higher by $\sim 0.3$, but with a negligible effect on the abundance ratios discussed here.

\subsection{Abundance determination}

Abundances for $\mathrm{Fe}, \mathrm{Na}, \mathrm{Al}, \mathrm{Si}, \mathrm{Ca}$, and $\mathrm{Ti}$ were derived from the equivalent widths of unblended transitions (measured with the code DAOSPEC) and using the code GALA (Mucciarelli et al. 2013a) based on the suite of software developed by R. L. Kurucz $^{1}$. Abundances for $\mathrm{Mg}, \mathrm{Ba}$, and $\mathrm{Eu}$ were derived from spectral synthesis because the Mg lines ( 6318-19 $\AA$ ) are located on the red wing of an auto-ionization $\mathrm{Ca}$ line, while the $\mathrm{Ba}$ and $\mathrm{Eu}$ lines are affected by isotopic and hyperfine splittings. Solar reference abundances are from Grevesse \& Sauval (1998). The line list and the determination of the abundance uncertainties are described in Appendices A and B, respectively.

\section{Chemical composition of Gaia1}

\subsection{Iron content}

Gaial has an average iron abundance of $[\mathrm{FeI} / \mathrm{H}]=+0.00 \pm$ $0.01 \mathrm{dex}(\sigma=0.03 \mathrm{dex})$. We used the maximum likelihood (ML) algorithm described in Mucciarelli et al. (2012) to estimate whether the observed scatter is compatible with a null intrinsic spread, taking into account the uncertainties of individual stars. The observed $[\mathrm{FeI} / \mathrm{H}]$ spread is fully consistent with a null intrinsic spread. The same conclusions are obtained when we adopt the pure spectroscopic and photometric sets of atmospheric parameters. The former set of parameters provides a very similar abundance, while the latter provides an average abundance lower by $\sim 0.2$ dex because of the lower $T_{\text {eff }}$.

S17 derived from 27 giant stars observed with HERMES an average abundance $[\mathrm{Fe} / \mathrm{H}]=-0.13 \pm 0.03 \mathrm{dex}(\sigma=0.13 \mathrm{dex})$. When we used the uncertainties they quoted, the ML algorithm suggests a non-null intrinsic spread, $\sigma_{\text {int }}=0.12 \pm 0.01 \mathrm{dex}$, which is at variance to our analysis. Even though their sample is significantly larger than ours, the intrinsic iron spread obtained from their abundances is likely due to two effects: (1) the large uncertainty in their spectroscopic parameters (see Fig. 1), and (2) the fact that their $[\mathrm{Fe} / \mathrm{H}]$ uncertainties do not include the contribution of the atmospherical parameter errors, hence they under-estimate the total error bar.

\section{2. $\alpha$-elements}

The measured $[\alpha / \mathrm{Fe}]$ abundance ratios (see Table C.1) are solarscaled, which indicates that the cluster formed from a gas enriched from both Type II and Type Ia supernovae. Figure C.1 shows the behaviour of the measured $[\alpha / \mathrm{Fe}]$ abundance ratios as a function of $[\mathrm{Fe} / \mathrm{H}]$ in comparison with the Galactic thin-disk stars (grey points, Soubiran \& Girard 2005). For all the abundance ratios, Gaial matches the mean locus described by the Galactic field stars well, suggesting a strong similarity with the Galactic thin disk.

\section{3. $\mathrm{Na}$ and $\mathrm{Al}$}

$\mathrm{Na}$ and $\mathrm{Al}$ are usually associated with nucleosynthesis by proton captures. We derived for Gaial $[\mathrm{Na} / \mathrm{Fe}]=-0.08 \pm 0.02$

\footnotetext{
1 http://kurucz.harvard.edu/programs.html, http://wwwuser. oats.inaf.it/castelli/sources.html
}

$(\sigma=0.05 \mathrm{dex})$ and $[\mathrm{Al} / \mathrm{Fe}]=+0.09 \pm 0.02(\sigma=0.05 \mathrm{dex})$. As visible in the upper panels of Fig. C.2, at the same metallicity of the cluster, the thin-disk stars are essentially solar-scaled and Gaia1 matches the observed Galactic trend well for both abundance ratios. We note that the $[\mathrm{Na} / \mathrm{Fe}]$ values shown in Fig. C.2 for Galactic stars do not include corrections for nonlocal thermodynamical equilibrium. In order to provide a homogeneous comparison with the literature data for the thin-disk stars, we did not take into account such corrections in our measured $[\mathrm{Na} / \mathrm{Fe}]$. However, corrections for the targets estimated according to Lind et al. (2011) are of about -0.1 dex, and they do not change our conclusions significantly.

No evidence of intrinsic $\mathrm{Na}$ and $\mathrm{Al}$ star-to-star scatters is found for these stars. Such chemical inhomogeneities are commonly observed among globular cluster stars (see Gratton et al. 2012, and references therein) and are usually explained within a framework of a self-enrichment process. On the other hand, open cluster stars, by virtue of their lower mass and density, do not undergo self-enrichment processes, and they show homogeneous $\mathrm{Na}$ and $\mathrm{Al}$ contents. The lack of significant star-tostar variations in Gaia1 (despite the small number of observed stars) agrees with its current low mass $\left(\sim 10^{4} M_{\odot}\right.$, Koposov et al. 2017). It is also likely that the cluster suffered only limited mass loss in the $\sim 3$ Gyr that elapsed since its formation.

\subsection{Neutron-capture elements: $\mathrm{Ba}$ and $\mathrm{Eu}$}

We measured $\mathrm{Ba}$, as prototype of elements that are produced through slow neutron captures, and $\mathrm{Eu}$, which is mainly produced through rapid neutron captures. We obtained $[\mathrm{Ba} / \mathrm{Fe}]=$ $+0.20 \pm 0.02(\sigma=0.05 \mathrm{dex})$ and $[\mathrm{Eu} / \mathrm{Fe}]=+0.04 \pm 0.03$ $(\sigma=0.08$ dex $)$, respectively. The lower panels of Fig. C. 2 show the behaviour of the $[\mathrm{Ba} / \mathrm{Fe}]$ and $[\mathrm{Eu} / \mathrm{Fe}]$ abundance ratios measured in Gaia1. For these two elements, we also find a good agreement with the Galactic thin-disk stars of similar metallicity.

\section{Conclusions}

The chemical composition of Gaia1 that we derived from MIKE spectra matches the composition of thin-disk stars and open clusters with similar metallicity well (see e.g. Pancino et al. 2010; Mishenina et al. 2015, and reference therein). This good match has been found for all the main groups of elements, that is, for $\alpha$ and proton- and neutron-capture elements, indicating that this cluster formed from a gas that was chemically enriched in a way similar to that of the Galactic thin disk. A possible extra-galactic origin of Gaial is not supported by the comparison between its chemical composition and that of other stellar systems. The galaxies currently populating the Local Group are more metal-poor than Gaial and do not reach solar metallicity (McConnachie 2012). The only extra-galactic environment approaching similar metallicities is the Sagittarius dwarf spheroidal galaxy, but its most metal-rich stars are characterized by sub-solar $[\alpha / \mathrm{Fe}]$ abundance ratios and an enhanced $[\mathrm{Ba} / \mathrm{Fe}]$ and $[\mathrm{Eu} / \mathrm{Fe}]$ (Bonifacio et al. 2000a; Monaco et al. 2005; Sbordone et al. 2007).

The mean metallicity of the thin disk (both field and open cluster stars) is known to decrease at large Galactocentric distances (Pancino et al. 2010; Hayden et al. 2015; Netopil et al. 2016; Magrini et al. 2017). As shown in Fig. 2, at the distance of Gaia1, the mean metallicity of open clusters is about -0.3 dex lower than that of the cluster, although a large dispersion is observed at these distances. Solar metallicity open clusters are typically found at $R_{\mathrm{GC}}$ of $\sim 7-8 \mathrm{kpc}$. On the other hand, stars with 


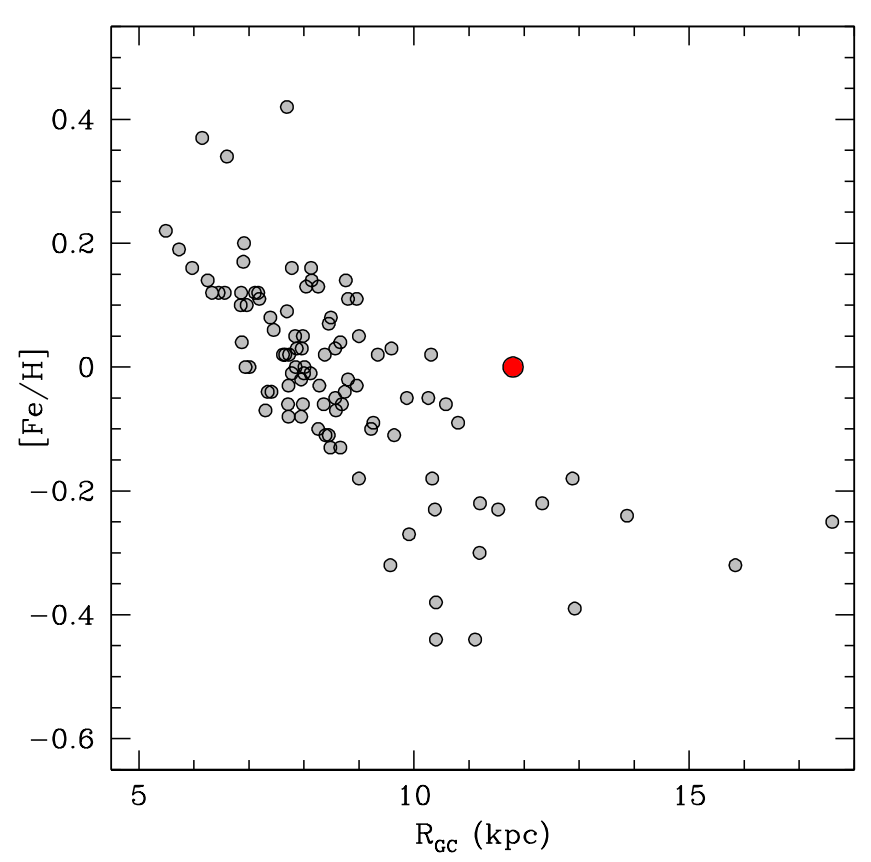

Fig. 2. Behaviour of $[\mathrm{Fe} / \mathrm{H}]$ as a function of the Galactocentric distance for open clusters (Netopil et al. 2016; Magrini et al. 2017, grey circles) and Gaia1 (red point).

solar metallicity are also present in the thin disk at similar distances: the metallicity distribution for thin-disk stars provided by Hayden et al. (2015) at $11<R_{\mathrm{GC}}<13 \mathrm{kpc}$ and $1<|z|<2 \mathrm{kpc}$ is peaked at $[\mathrm{Fe} / \mathrm{H}]=-0.38 \mathrm{dex}$, but it reaches super-solar metallicities.

Our results on the chemical composition strongly argue against an extra-galactic origin for Gaia1. It appears to be an unremarkable standard Galactic open cluster. Its position with respect to the overall trend between $[\mathrm{Fe} / \mathrm{H}]$ and $R_{\mathrm{GC}}$ could suggest that it formed in the inner disk, progressively migrating toward higher $R_{\mathrm{GC}}$, thus explaining its possible peculiar orbit with respect to other open clusters. However, it is worth noting that the precise value of $R_{\mathrm{GC}}$ can be affected by the value of $E(B-V)$, which still remains uncertain for this cluster. We compared the measured abundance ratios with those derived by Nissen et al. (2016) for solar twin stars of ages similar to that of Gaial in the solar neighbourhood. Within the uncertainties, the abundances of Gaia1 are comparable with those of the solar neighbourhood, except for $[\mathrm{Al} / \mathrm{Fe}]$ and $[\mathrm{Ba} / \mathrm{Fe}]$, which in this cluster are higher (by $\sim 0.1$ dex) with respect to coeval solar twin stars. However, we cannot entirely rule out that Gaia1 originally formed in the solar neighbourhood.

Although the orbital parameters inferred by S17 may suggest that the cluster has been accreted by the Milky Way, they are still, within the uncertainties, fully compatible with the majority of known Galactic open clusters. The uncertainty on the orbit of Gaial is dominated by the uncertainty on its proper motion. The situation will be greatly improved with the second Gaia data release, and we defer any conclusion on its kinematics to that time.

Acknowledgements. We thank the anonymous referee for the useful comments and suggestions. We are grateful to P. Di Matteo for useful discussions on the Galactic metallicity gradient. L.M. acknowledges support from "Proyecto interno" of the Universidad Andres Bello. P.B. acknowledges financial support from the Scientific Council of Observatoire de Paris and from the action fédératrice "Exploitation Gaia".

\section{References}

Bensby, T., Feltzing, S., Lundstrom, I., \& Ilyin, I. 2005, A\&A, 433, 185 Bernstein, R., Shectman, S. A., Gunnels, S. M., Mochnacki, S., \& Athey, A. E. 2003, Proc. SPIE, 4841, 1694

Bonifacio, P., Hill, V., Molaro, P., et al. 2000a, A\&A, 359, 663

Bonifacio, P., Monai, S., \& Beers, T. C. 2000b, AJ, 120, 2065

Bressan, A., Marigo, P., Girardi, L., et al. 2012, MNRAS, 427, 127

Buzzoni, A., Patelli, L., Bellazzini, M., Fusi Pecci, F., \& Oliva, E. 2010, MNRAS, 403, 1592

Kelson, D. D. 2003, PASP, 115, 688

Koposov, S. E., Belokurov, V., \& Torrealba, G. 2017, MNRAS, in press [arXiv: 1702.01122]

Fuhr, J. R., \& Wiese, W. L. 2006, JPCRD, 35, 1669

Gaia Collaboration (Brown, A. G. A., et al.) 2016, A\&A, 595, A2

Garz, T. 1973, A\&A, 26, 471

Gonzalez Hernandez, J. I., \& Bonifacio, P. 2009, A\&A, 497, 497

Gratton, R. G., Carretta, E., \& Bragaglia, A. 2012, A\&ARv, 20, 50

Grevesse, N., \& Sauval, A. J. 1998, Space Sci. Rev., 85, 161

Hayden, M. R., Bovy, J., Holtzman, J. A., et al. 2015, ApJ, 808, 132

Lawler, J. E., Wickliffe, M. E., den Hatog, E. A., \& Sneden, C. 2001, ApJ, 563, 1075

Lawler, J. E., Guzman, A., Wood, M. P., Sneden, C., \& Cowan, J. J. 2013, ApJS, 205,11

Lind, K., Asplund, M., Barklem, P., \& Belyaev, A. K. 2011, A\&A, 528, A103

Magrini, L., Randich, S., Kordopatis, G., et al. 2017, A\&A, 603, A2

Martin, G. A., Fuhr, J. R., \& Wiese, W. L. 1988, New York: American Institute of Physics (AIP) and American Chemical Society, 1988

McConnachie, A. 2012, AJ, 144, 4

Melendez, J., \& Barbuy, B. 2009, A\&A, 497, 611

Mishenina, T., Pignatari, M., Carraro, G., et al. 2015, MNRAS, 446, 3651

Monaco, L., Bellazzini, M., Bonifacio, P., et al. 2005, A\&A, 441, 141

Mucciarelli, A., Bellazzini, M., Ibata, R., et al. A. 2012, MNRAS, 426, 2889

Mucciarelli, A., Pancino, E., Lovisi, L., Ferraro, F. R., \& Lapenna, E. 2013, ApJ, 766,78

Neckel, H., \& Labs, D. 1984, Sol. Phys., 90, 205

Netopil, M., Paunzen, E., Heiter, U., \& Soubiran, C. 2016, A\&A, 585, A150

Nissen, P. E. 2016, A\&A, 593, A65

Pancino, E., Carrera, R., Rossetti, E., \& Gallart, C. 2010, A\&A, 511, A56

Reddy, B. E., Tomkin, J., Lambert, D. L., \& Allende Prieto, C. 2003, MNRAS, 340,304

Sbordone, L., Bonifacio, P., Buonanno, R., et al. 2007, A\&A, 465, 815

Schlegel, D. J., Finkbeiner, D. P., \& Davis, M. 1998, ApJ, 500, 525

Simpson, J. D., De Silva, G. M., Martell, S. L., et al. 2017, MNRAS, submitted [arXiv: 1703.03823]

Smith, G., \& Raggett, D. St. J. 1981, J. Phys. B, 14, 4015

Soubiran, C., \& Girard, P. 2005, A\&A, 438, 139

Stetson, P. B., \& Pancino, E. 2008, PASP, 120, 1332 
Table A.1. Wavelength, oscillator strength, excitation potential, and measured EW for the transitions.

\begin{tabular}{cccccc}
\hline \hline ID & $\begin{array}{c}\lambda \\
(\AA)\end{array}$ & Element & $\log g f$ & $\begin{array}{c}\chi W \\
(\mathrm{eV})\end{array}$ & $\begin{array}{c}E W \AA) \\
(\mathrm{m})\end{array}$ \\
\hline 1 & 5197.9 & 26.00 & -1.620 & 4.300 & 45.30 \\
1 & 5236.2 & 26.00 & -1.497 & 4.190 & 71.10 \\
1 & 5249.1 & 26.00 & -1.460 & 4.470 & 47.60 \\
1 & 5315.1 & 26.00 & -1.550 & 4.370 & 55.10 \\
1 & 5326.8 & 26.00 & -2.100 & 4.410 & 25.60 \\
1 & 5379.6 & 26.00 & -1.514 & 3.690 & 83.20 \\
1 & 5386.3 & 26.00 & -1.740 & 4.150 & 45.90 \\
1 & 5398.3 & 26.00 & -0.710 & 4.450 & 96.60 \\
1 & 5405.4 & 26.00 & -1.390 & 4.390 & 69.60 \\
1 & 5412.8 & 26.00 & -1.716 & 4.430 & 40.00 \\
1 & 5470.1 & 26.00 & -1.790 & 4.450 & 33.30 \\
1 & 5476.3 & 26.00 & -0.935 & 4.140 & 100.60 \\
1 & 5491.8 & 26.00 & -2.188 & 4.190 & 34.40 \\
1 & 5522.4 & 26.00 & -1.520 & 4.210 & 71.90 \\
1 & 5525.5 & 26.00 & -1.084 & 4.230 & 87.20 \\
1 & 5543.9 & 26.00 & -1.110 & 4.220 & 92.60 \\
1 & 5577.0 & 26.00 & -1.550 & 5.030 & 25.40 \\
1 & 5609.0 & 26.00 & -2.400 & 4.210 & 14.30 \\
\hline
\end{tabular}

Notes. Complete version of the table is available in electronic form.

\section{Appendix A: Line list}

The analysed lines were selected according to a synthetic spectrum calculated with the code SYNTHE, adopting a model atmosphere calculated with ATLAS9 with the representative atmospheric parameters of the stars (that have $T_{\mathrm{eff}}, \log g$ and $v_{t}$ very similar each other). The reference synthetic spectrum was computed including all the atomic and molecular transitions of the last version of the Kurucz and Castelli line lists ${ }^{2}$, updating the oscillator strengths for some transitions of interest (as explained below). For the abundances calculated using the equivalent widths (EW), we selected only lines that are predicted to be unblended. Atomic data for the Fe I lines are from Martin et al. (1998) and Fuhr \& Wiese (2006), while those for the Fe II lines are from Melendez \& Barbuy (2009). Oscillator strengths for the Ca I lines are mainly from Smith \& Raggett (1981), for the Ti I lines they are from Martin et al. (1998) and Lawler et al. (2013). For the Si I lines we adopted, when available, the furnace oscillator strengths by Garz (1973), while for other lines, for which the available $\log g f$ have large uncertainties or only poorly reproduce the solar spectrum, we derived astrophysical oscillator strengths using the solar flux spectra of Neckel \& Labs (1984) and a solar model atmosphere calculated with the chemical mixture of Grevesse \& Sauval (1998). The same method was adopted to compute the oscillator strengths for the Al I doublet at 6696-6698 $\AA$ because the available values in the literature underestimate the solar abundance by about 0.2 dex. For the two Na I doublets at 5682-88 $\AA$ and 6154-6160 $\AA$ we employed the $\log g f$ available in the NIST database ${ }^{3}$, which we also used for the Mg I doublet at 6318-6319 А. For the transitions measured using spectral synthesis because they are affected by hyperfine and isotopic splitting, we adopted the line lists available in the Kurucz and Castelli database (Ba II lines) and the line list provided by Lawler et al. (2001; Eu II line). Table A.1 lists the measured EW for all the used lines together with the adopted oscillator strength and excitation potential.

\section{Appendix B: Uncertainties}

The total uncertainty in the measured $[X / Y]$ abundance ratio was computed by adding two terms in quadrature.

1. The error related to the line measurement. For elements whose abundance was derived through the measured EWs, this term was estimated as the dispersion of the mean divided by the root mean square of the number of lines. For $\mathrm{Mg}$, Ba, and $\mathrm{Eu}$, for which we used spectral synthesis, this error was estimated using Monte Carlo simulations, injecting Poissonian noise into the best-fit spectrum in order to reproduce the measured $\mathrm{S} / \mathrm{N}$, and creating a sample of 500 noisy synthetic spectra for each line. These spectra were re-analysed with the same procedure as was used for the observed lines and the $1 \sigma$ dispersion of the abundance distribution taken as uncertainty.

2. The error related to the atmospheric parameters. This uncertainty was calculated by varying each time only one parameter by the corresponding error, keeping them fixed, and repeating the analysis. Uncertainties in spectroscopic $T_{\text {eff }}$ and $v_{\mathrm{t}}$ were estimated according to the error in the slope between excitation potential and iron abundances, and between reduced $\mathrm{EW}(\log (E W / \lambda))$ and iron abundances (see Mucciarelli et al. 2013a, for details). Uncertainties in surface gravities were computed by including the errors in spectroscopic $T_{\text {eff }}$, mass, reddening, and distance modulus. 


\section{Appendix C: Derived chemical abundances}

Table C.1. Measured chemical abundance ratios for individual MIKE targets.

\begin{tabular}{|c|c|c|c|c|c|c|c|c|c|}
\hline ID & {$[\mathrm{FeI} / \mathrm{H}]$} & {$[\mathrm{Na} / \mathrm{Fe}]$} & {$[\mathrm{Mg} / \mathrm{Fe}]$} & {$[\mathrm{Al} / \mathrm{Fe}]$} & {$[\mathrm{Si} / \mathrm{Fe}]$} & {$[\mathrm{Ca} / \mathrm{Fe}]$} & {$[\mathrm{Ti} / \mathrm{Fe}]$} & {$[\mathrm{BaII} / \mathrm{Fe}]$} & [EuII/Fe] \\
\hline 1 & $02 \pm 0.07$ & $-0.14 \pm 0.05$ & $+0.02 \pm 0.04$ & $+0.13 \pm 0.05$ & $+0.01 \pm 0.07$ & $-0.03 \pm 0.05$ & $+0.02 \pm 0.06$ & $+0.26 \pm 0.10$ & $-0.04 \pm 0.06$ \\
\hline 2 & & 77 & & & & & & & \\
\hline 4 & $-0.02 \pm$ & $-0.13 \pm$ & $+0.09=$ & +0.08 & -0.02 & -0.08 & +0.00 & +0.2 & -0 . \\
\hline 5 & $+0.00 \pm 0.06$ & $-0.08 \pm 0.05$ & $+0.00 \pm 0.05$ & $+0.13 \pm 0.04$ & $-0.01 \pm 0.07$ & -0.09 & -0.04 & +0.2 & +0.1 \\
\hline 6 & & -0.0 & & & & & & & \\
\hline 7 & $-0.03 \pm 0.06$ & $-0.02 \pm 0.06$ & $+0.11 \pm 0.07$ & $+0.11 \pm 0.04$ & $-0.03 \pm 0.07$ & $-0.01 \pm 0.05$ & $+0.02 \pm 0.06$ & $+0.15 \pm 0.11$ & $+0.07 \pm 0.07$ \\
\hline Mean $(\sigma)$ & $+0.00(0.03)$ & $-0.08(0.05)$ & $+0.04(0.05)$ & $+0.09(0.04)$ & $+0.00(0.03)$ & $-0.05(0.03)$ & $-0.01(0.03)$ & $+0.20(0.05)$ & $+0.04(0.08)$ \\
\hline
\end{tabular}

Notes. The last row lists the mean abundances and the corresponding $1 \sigma$ uncertainty.

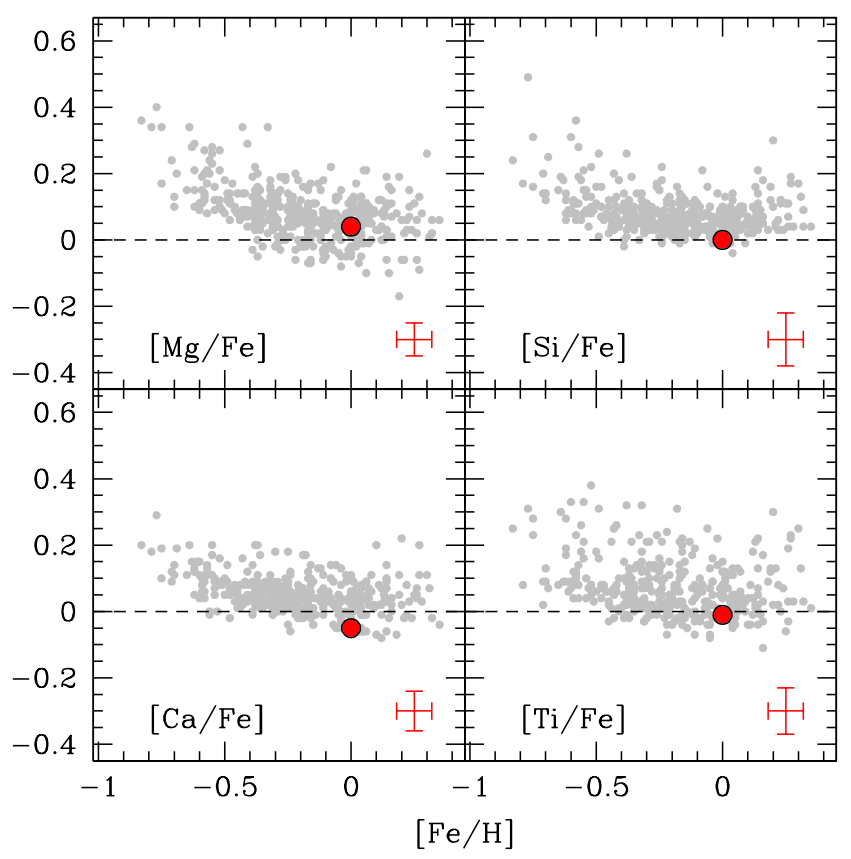

Fig. C.1. Behaviour of the average $[\mathrm{Mg} / \mathrm{Fe}],[\mathrm{Si} / \mathrm{Fe}],[\mathrm{Ca} / \mathrm{Fe}]$, and [Ti/Fe] abundance ratios of Gaial as a function of $[\mathrm{Fe} / \mathrm{H}]$ (red point) in comparison with Galactic thin-disk stars (grey points, Soubiran \& Girard 2005).

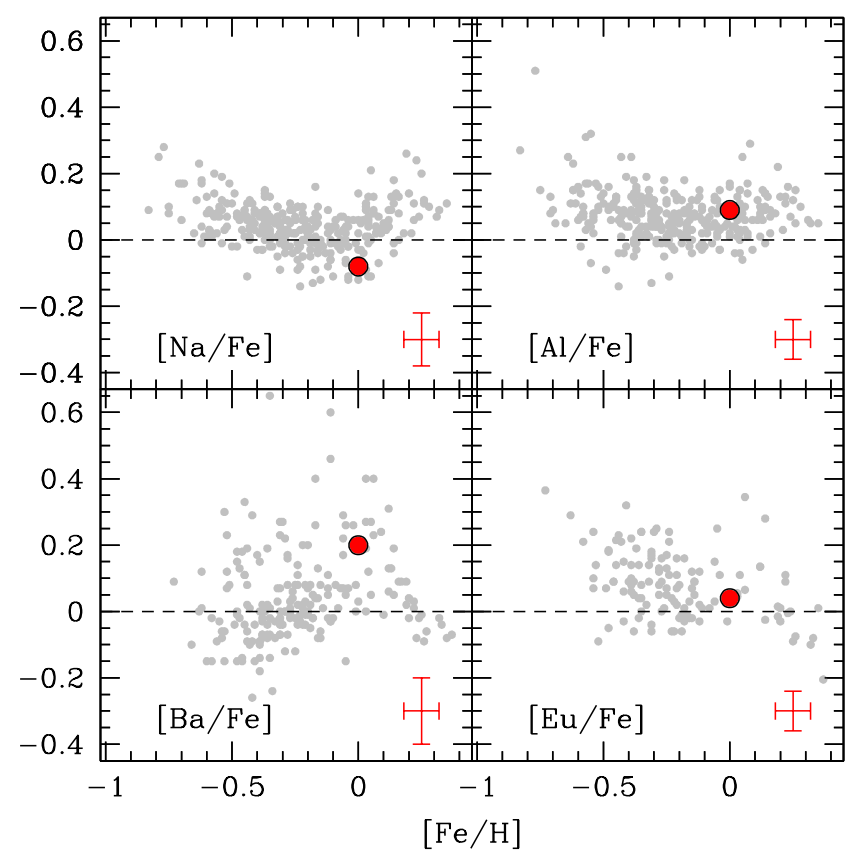

Fig. C.2. Behaviour of the average $[\mathrm{Na} / \mathrm{Fe}],[\mathrm{Al} / \mathrm{Fe}],[\mathrm{Ba} / \mathrm{Fe}]$, and $[\mathrm{Eu} / \mathrm{Fe}]$ abundance ratios of Gaial as a function of $[\mathrm{Fe} / \mathrm{H}]$ (red point) in comparison with Galatic thin-disk stars (grey points, Soubiran \& Girard (2005) for Na and Al, and Reddy et al. (2003), Bensby et al. (2005) for $\mathrm{Ba}$ and $\mathrm{Eu}$. 